\title{
An Optimal Estimation Retrieval Algorithm for Microwave Humidity Sounding Channels with Minimal Scan Position Bias
}

\author{
RichARD M. SCHULTE AND CHRISTIAN D. KUMMEROW \\ Department of Atmospheric Science, Colorado State University, Fort Collins, Colorado
}

(Manuscript received 31 July 2018, in final form 17 December 2018)

\begin{abstract}
A flexible and physical optimal estimation-based inversion algorithm for retrieving atmospheric water vapor and cloud liquid water path from passive microwave radiometers over the global oceans is presented. The algorithm's main strength lies in its ability to explicitly account for forward model errors that depend on the Earth incidence angle (EIA) at which a given radiometer measurement is made. Validation of total precipitable water (TPW) retrieved from Microwave Humidity Sounder (MHS) measurements against nearcoincident estimates of TPW from SuomiNet GPS ground stations shows that retrieved TPW values agree closely with SuomiNet estimates, and somewhat better than values from the Microwave Integrated Retrieval System that are retrieved from the same MHS instruments. More importantly, it is found that the inclusion of appropriate forward model error assumptions, which are tailored to the EIA and sea surface temperature of the scene being considered, are able to almost entirely eliminate EIA-dependent biases in retrieved TPW. This result holds true across all satellites currently carrying an MHS instrument, despite the fact that only measurements from one satellite are used to estimate forward model errors. The consistency achieved by the retrieval algorithm across all view angles suggests that other inversion algorithms, particularly those for crosstrack-scanning radiometers and potential future constellations of small satellites, would benefit from the inclusion of nuanced error assumptions that consider the effect of EIA.
\end{abstract}

\section{Introduction}

Water vapor and clouds are fundamentally important to Earth's energy budget and climate system. Water vapor is a potent greenhouse gas, and it is widely postulated that increases in the global temperature should be linked to increases in the amount of water vapor in the atmosphere (Mears et al. 2007; O'Gorman and Muller 2010). It is thus important to a have a global, long-term, reliable record of total column water vapor, or total precipitable water (TPW), for monitoring climate change. Knowledge of global water vapor fields is also of great interest to operational weather forecasters (Forsythe et al. 2015). Likewise, clouds have radiative effects that strongly influence regional and global climate, and cloud-related feedbacks are the largest source of uncertainty in climate model projections (Boucher et al. 2013). Clouds also link atmospheric and hydrologic processes, transfer moisture and momentum, and affect large-scale circulations through latent heat release. Global measurements of cloud water

Corresponding author: Richard Schulte, rschulte@atmos. colostate.edu and ice content are thus an important part of a holistic understanding of the hydrologic cycle.

Passive microwave radiometers have been used for nearly three decades to measure atmospheric water vapor over the global oceans. Historically, passive microwave radiometers have usually been classified as either "imagers" or "sounders." Imagers are generally conically scanning and typically feature several channels with sensitivity to the surface, while sounders tend to be cross-track scanning and tend to have less surface sensitivity but more information about the vertical distribution of water vapor. Retrieval of atmospheric parameters from crosstrack-scanning instruments presents some difficulties not applicable to conically scanning ones. Namely, while imagers maintain a constant Earth incidence angle (EIA), the EIA for a sounder changes across each scan, meaning that the slant path through the atmosphere, as well as the instantaneous field of view (IFOV), changes with each pixel. It is desirable, of course, that changes in the atmospheric parameters retrieved by sounders from one pixel to another should be entirely attributable to differences in the atmospheric state, with no EIA-induced bias. This could become especially important over the next decade, as 
constellations of more cost-efficient small satellites offer the possibility of achieving global coverage from passive microwave sounders with temporal resolution of less than one hour (Blackwell et al. 2018; Ma et al. 2017). Others propose using a train of closely spaced CubeSats to observe the evolution of clouds on even shorter time frames of only a few minutes (Reising et al. 2015). In any case, in order for a constellation of sounders to be able to reliably give information about changes in atmospheric state, any uncertainty in retrieved parameters related to changes in the EIA from one satellite observation to another must be proven to be small in comparison to the actual magnitude of changes that are possible over the size of the instrument FOV.

Both imagers and sounders have been used for several decades to estimate TPW over the global oceans, and in recent years many retrieval algorithms have been put forth (e.g., Boukabara et al. 2010; Duncan and Kummerow 2016, hereafter DK16; Mears et al. 2015) that agree reasonably well with observations from radiosondes and groundbased measurements from global positioning system (GPS) stations. These algorithms, however, have limitations. For example, some algorithms are sensor specific or, even if not limited to a single instrument, are limited to either imagers or sounders. Algorithms applicable to both conically and cross-track-scanning instruments allow for more frequent observations than those considering only one category of instrument, and can ensure greater consistency than could be achieved by relying upon two separate algorithms. In addition, such algorithms are useful for calibrating cross-track instruments with respect to larger, more expensive, and yet more trusted conically scanning instruments such as the Global Precipitation Measurement (GPM) Microwave Imager (GMI). The Microwave Integrated Retrieval System (MiRS; Boukabara et al. 2011) is one such algorithm than can be used with both sounders and imagers, and it has the added benefit of retrieving values over land. However, some MiRS-retrieved parameters, notably TPW, are known to exhibit biases toward the edge of scan for sounding instruments, where EIA values are large (Boukabara et al. 2010). In this paper, we present our own retrieval algorithm applicable to both conically scanning and cross-track-scanning passive microwave radiometers. Specially, we evaluate retrieved values of oceanic TPW and cloud liquid water path (LWP) from Microwave Humidity Sounder (MHS) instruments, although the retrieval methodology is flexible enough to be applied to virtually any passive microwave radiometer. TPW values are validated against estimates from GPS ground stations, and both TPW and LWP values are compared to corresponding retrieved values from MiRS, which uses the same underlying radiance measurements. Like MiRS, our algorithm is based on optimal estimation
(OE) theory. It produces values that are in reasonable agreement overall with MiRS, but that also demonstrate markedly less bias at high view angles, suggesting a way that similar physically based retrieval algorithms for microwave sounders might be improved.

Our data sources are explained more thoroughly in section 2. In section 3, we describe the retrieval algorithm and focus especially on the component of the algorithm that we believe to be key in reducing EIA-related biases: the forward model error covariance matrix. In section 4 we present the results of our comparisons, and section 5 offers some commentary and thoughts on future applications of this work.

\section{Data}

The MHS is a five-channel, passive microwave radiometer operating at frequencies near $89,157,183.3 \pm 1$, $183 \pm 3$, and $190.3 \mathrm{GHz}$. It is a cross-track-scanning radiometer with an IFOV of $1.1^{\circ}$ (corresponding to a bestcase spatial resolution of about $17 \mathrm{~km}$ at nadir) and an overall swath width of approximately $1920 \mathrm{~km}\left( \pm 49.5^{\circ}\right)$. MHS instruments are on board the MetOp-A, MetOp-B, $N O A A-18$, and $N O A A-19$ spacecraft. These satellites are all in sun-synchronous orbits with inclinations near $99^{\circ}$. In this study, we use level-1C (L1C) intercalibrated brightness temperatures $\left(T_{b} \mathrm{~s}\right)$ that were developed by the GPM mission Intersatellite Calibration Working Group (XCAL; Berg et al. 2016).

Ancillary data (surface wind speeds, surface pressures, and temperature profiles) used by the retrieval algorithm are taken from the European Centre for Medium-Range Weather Forecasts (ECMWF) reanalysis product, ERA5 (ECMWF 2017). The reanalysis data have a temporal resolution of $1 \mathrm{~h}$ and a horizontal spatial resolution of $30 \mathrm{~km}$, with 37 standard pressure levels that are subset to match the coarser vertical resolution of the forward model. ERA5 data are also used to determine the a priori water vapor profile. Sea surface temperature (SST) information comes from the Reynolds et al. (2007) Optimum Interpolation Sea Surface Temperature (OISST) product, and sea surface salinity is taken from Aquarius monthly climatologies (Le Vine et al. 2015).

Several data sources are used for validation and comparison. Retrieved values are compared against corresponding values for the same satellites from MiRS. MiRS orbital level-2 output is obtained from the NOAA Comprehensive Large Array-Data Stewardship System (CLASS). The MiRS data analyzed in this study were produced in 2016, corresponding to version 9.2 of the algorithm. In 2017, operational production switched to version 11.2 which included a number of enhancements, including retrievals at higher MHS resolution, use of 
a newer version (2.1.1) of the Community Radiative Transfer Model (CRTM) forward model, and updated radiometric bias corrections. These changes resulted in smaller, but still significant, scan-dependent biases in retrieved TPW. It is worth noting that all satellites carrying an MHS sensor also have an Advanced Microwave Sounding Unit-A (AMSU-A), and that radiances from this instrument are also ingested into the MiRS algorithm. AMSU-A has 15 channels at frequencies ranging from 23.8 to $89.0 \mathrm{GHz}$, and is primarily used for temperature sounding. It also has an IFOV that is much larger than MHS, with roughly nine MHS pixels per AMSU-A pixel. Because of this IFOV discrepancy, and because most of the channels are best used for temperature sounding, we do not consider these radiances in our retrieval algorithm, though they could easily be added into the OE framework. Version 9.2 MiRS orbital data are offered at MHS resolution for MetOp-B, but only at AMSU-A resolution for the other satellites. Thus, for MetOp-A, NOAA-18, and NOAA-19, our retrieved values are first averaged to match the MiRS resolution before comparisons are made.

Retrieved values are also compared to estimates from the Modern-Era Retrospective Analysis for Research and Applications, version 2 (MERRA-2; Gelaro et al. 2017). Retrieved TPW from both MiRS and the OE algorithm presented in this paper, along with MERRA-2 TPW values, are validated against estimates of TPW derived from zenith path delay at SuomiNet GPS stations around the globe (Ware et al. 2000). The methodology by which these estimates are calculated is laid out in Wang et al. (2007). They estimated total errors to be less than $1.5 \mathrm{~mm}$.

\section{Methods}

Many early passive microwave retrieval algorithms were regression-based (e.g., Alishouse et al. 1990; Wilheit and Chang 1980) or semiphysical methods that made assumptions to simplify the equations of radiative transfer in the atmosphere to make the inverse problem more tractable (Greenwald et al. 1993; Wentz 1997). More recently, retrieval algorithms have been developed that make use of fully physical forward models, allow for the retrieval of all parameters of interest simultaneously, and are not tied to a specific sensor or set of frequencies. Algorithms of this type are better able to ensure that the retrieved atmospheric parameters are consistent with each other across sensors and that, when put into a forward model, result in reasonable simulated brightness temperatures. Sensorindependent algorithms are desirable in that they allow for the creation of consistent records of geophysical variables across the ever-expanding history of spaceborne passive microwave radiometers. A common approach is to iteratively solve for a collection of geophysical parameters by repeatedly forward modeling the transfer of radiation through the atmosphere until a solution for the atmospheric state vector is found that yields forward modeled $T_{b} \mathrm{~s}$ close to those observed by the satellite while also being consistent with prior knowledge (a priori information) about the state of the atmosphere (Boukabara et al. 2011; Deblonde and English 2003; Elsaesser and Kummerow 2008). The Colorado State University $1 \mathrm{D}$ variational data assimilation (CSU 1DVAR) retrieval algorithm (DK16) is one such algorithm, having initially been developed for the retrieval of nonraining parameters for GMI but having been adapted for use with other conically scanning instruments. Here we demonstrate that the CSU 1DVAR algorithm can also be used to retrieve information from cross-track sounders.

\section{a. CSU 1DVAR}

The 1DVAR technique, also known as optimal estimation, is a regularized matrix inverse method based on Bayes's theorem. The mathematics of the method is laid out explicitly in Rodgers (2000), but the most important parts are reviewed here for ease in understanding the results that follow. The relationship between the physical state of the atmosphere and the $T_{b}$ s measured by a passive microwave radiometer can be generalized by

$$
\mathbf{y}=f(\mathbf{x}, \mathbf{b})+\varepsilon,
$$

where $\mathbf{y}$ is the measurement vector containing the observed $T_{b}$ s at each radiometer channel and $\mathbf{x}$ is the state vector containing the atmospheric properties to be estimated. For the purposes of this paper, the state vector contains five parameters: the three leading principal components (PCs) of the water vapor profile, the cloud LWP, and the ice water path (IWP). Since optimal estimation involves an assumption of Gaussian-distributed parameters, and the distributions of LWP and IWP more closely resemble lognormal distributions, the logarithms of these parameters are retrieved instead. Note that surface wind speed is also included in the state vector for conically scanning radiometers. In addition to having window channels that are better able to see the surface, these instruments measure both vertically and horizontally polarized radiation, allowing for the separation of the wind speed signal from SST and atmospheric signals. To calculate simulated $T_{b}$ s, the forward model $f$ depends not only on the parameters in the state vector but also on a variety of parameters not solved for but assumed to be known in the model atmosphere (e.g., surface temperature, surface wind speed, vertical temperature profile, cloud height and depth). These ancillary parameters 
are accounted for in the vector $\mathbf{b}$. Finally, $\boldsymbol{\varepsilon}$ is an error term containing uncertainties due to sensor noise, errors in the forward model, and uncertainties in the forward model parameter assumptions $(\mathbf{b})$. The forward modeled $T_{b} \mathbf{s} f(\mathbf{x}, \mathbf{b})$ should agree with the satellite measurements $\mathbf{y}$ within the model and sensor error estimates given by $\boldsymbol{\varepsilon}$. The aim of the 1DVAR algorithm is to find the most likely state vector $\mathbf{x}$, given measurements $\mathbf{y}$, prior knowledge about the state of the atmosphere, and proper error estimates.

Due to the ill-posed and underconstrained nature of the inverse problem, an a priori state vector $\mathbf{x}_{a}$ is used to prevent the retrieval algorithm from settling on a solution that fits the observed brightness temperatures but is physically implausible. The water vapor component of $\mathbf{x}_{a}$ is taken from ERA5 reanalysis. On the other hand, for all pixels, the a priori values for LWP and IWP are set to the nominally low value of $10 \mathrm{~g} \mathrm{~m}^{-2}$. This helps ensure that significant cloud liquid or ice is only retrieved when there is a clear radiometric signal suggestive of the presence of clouds. The assumed errors in the a priori parameters, and their covariances, are described by the a priori error matrix $\mathbf{S}_{a}$. Another error covariance matrix, the $\mathbf{S}_{y}$ matrix, gives the errors assumed for each radiometer channel as the main diagonal elements of the matrix and the covariances of the errors between the channels as the off-diagonal elements. Within this formalism, assuming that both the parameters in $\mathbf{x}$ and the statistics of the measurements themselves are Gaussian, it can be shown (Rodgers 2000) that finding the most likely state vector amounts to minimizing the cost function $\Phi$ :

$$
\begin{aligned}
\Phi= & \left(\mathbf{x}-\mathbf{x}_{a}\right)^{\mathrm{T}} \mathbf{S}_{a}^{-1}\left(\mathbf{x}-\mathbf{x}_{a}\right) \\
& +[\mathbf{y}-f(\mathbf{x}, \mathbf{b})]^{\mathrm{T}} \mathbf{S}_{y}^{-1}[\mathbf{y}-f(\mathbf{x}, \mathbf{b})] .
\end{aligned}
$$

The cost function thus weights both measurements and prior knowledge, in relation to their uncertainties. Minimizing $\Phi$ and finding the maximum probability state vector $\mathbf{x}$ is accomplished by finding the value for $\mathbf{x}$ at which the gradient of the cost function $\nabla_{x} \Phi$ is equal to zero. The Gauss-Newton method is used to solve iteratively for this value, with convergence achieved once there is very little change between iterations [Eq. (5.29) in Rodgers 2000].

\section{b. Forward model description}

The forward model assumes a 16-layer atmosphere with pressures ranging from $100 \mathrm{hPa}$ to the surface. The depth of each layer is fixed, except for the lowest layer, which varies according to the sea level pressure specified by ERA5. Other values taken from reanalysis are the wind speed and direction (needed to compute surface emissivities), and the atmospheric temperature profile, because the microwave frequencies employed by MHS and similar satellites do not contain enough information to be able to reliably solve for these parameters. The forward model uses the Monochromatic Radiative Transfer Model (MonoRTM; Clough et al. 2005) to calculate absorption coefficients for each layer, and the FASTEM6 model (Kazumori and English 2015) is used to determine the ocean surface emissivity.

It is not feasible to reliably and independently retrieve a full 16-level water vapor profile from only a handful of radiometer channels (five, in the case of MHS). Thus, following the example of other retrieval algorithms such as DK16 or MiRS, the three leading PCs of the water vapor profile are retrieved instead. This approach makes use of the fact that water vapor profiles tend to have similar shapes and that the water vapor content of one atmospheric level will tend to be correlated with the water vapor content of the levels above and below it. The PCs are defined as deviations from a mean profile, and both the mean profile and the PCs are calculated from ERA5 reanalysis. We subset the mean profiles and PCs by SST (in 1-K bins) because water vapor mixing ratios, especially in the lower levels of the atmosphere, are strongly correlated with temperature. This subsetting allows the forward model to capture much more (approximately 90\%) of the natural water vapor profile variability with just the three leading PCs than would be possible otherwise.

All cloud water is assumed to be distributed evenly between the pressure levels of 800 and $925 \mathrm{hPa}$ (or $900 \mathrm{hPa}$, if the SST is below $280 \mathrm{~K}$ ). Various other methods of distributing the cloud water in the forward model were tested; however, unless reliable a priori information about the heights and depths of the clouds is known a priori, the overall effect on the cloud water retrieval is small. Errors in the simulated $T_{b}$ s caused by this simplistic representation of liquid clouds are somewhat mitigated by the fact that the emissivity of cloud drops increases with decreasing temperature (Mätzler et al. 2010), meaning that the emission from the cloud is tied more closely to the total amount of liquid water than it is to cloud height. A monodisperse drop size distribution (DSD) of spherical cloud droplets of radius $12 \mu \mathrm{m}$ is assumed. This should not be a major cause for concern because most nonprecipitating cloud droplets are small enough that scattering is negligible and absorption is largely dependent on the total mass of water in the cloud, not the DSD (Bennartz 2007).

Greater uncertainty comes from the representation of cloud ice in the forward model. Most of the effect on brightness temperatures due to ice comes from 
scattering, rather than absorption. We have chosen the IWP (i.e., the amount of ice) to be the free parameter retrieved by the 1DVAR algorithm, necessitating the specification of a variety of other ice-related parameters that we acknowledge carry large uncertainties. For example, assumptions made in regard to both ice crystal habit and size distribution can have a large effect on forward modeled $T_{b}$ s. (Kulie et al. 2010). We consider the IWP part of the CSU 1DVAR state vector to mostly be a way for the forward model to account for scattering from ice and thus allow for convergence in a greater number of scenes. IWP estimates are notoriously difficult to validate and the evaluation of CSU 1DVAR retrieved IWP values is beyond the scope of this paper. Thus, we make assumptions that we believe are reasonable, yet not overly complex.

As with liquid clouds, the ice cloud level is fixed, in this case with all cloud ice being assumed to reside between 300 and $400 \mathrm{hPa}$. This level was chosen so as to minimize differences between simulated and observed $T_{b} \mathrm{~S}$ when state vectors were taken from ERA5 reanalysis to forward model $T_{b} \mathrm{~s}$ at a representative sample of MHS pixels. Since simulated $T_{b}$ s also depend strongly on particle size and shape, this method of distributing cloud ice could very well be suboptimal. However, given the large uncertainties in ice particle size and shape, a more sophisticated method of distributing cloud ice would add computational complexity without necessarily reducing the uncertainty in the retrieved IWP.

The ice particle size distribution used in the algorithm comes from Field et al. (2007). Ice particles are assumed to have a constant density of $100 \mathrm{~kg} \mathrm{~m}^{-3}$, and particle mass is assumed to be proportional to $D^{3}$, where $D$ is the maximum dimension of the particle. To calculate the scattering properties of the ice particles, the forward model makes use of a database of single-scattering properties at microwave frequencies for various ice crystal habits (Liu 2008) as well as an associated database for larger aggregates of ice crystals (Nowell et al. 2013). These databases use the discrete dipole approximation method (e.g., Draine and Flatau 1994) to compute scattering properties by approximating a continuum target with a finite array of polarizable points. As for particle shape, the forward model assumes 6-bullet rosettes (database shape number 8) for particles with a maximum dimension less than $800 \mu \mathrm{m}$ and aggregates of $400-\mu \mathrm{m}$ rosettes (shape 12) for particles $800 \mu \mathrm{m}$ or larger.

\section{c. Error covariance matrices}

The matrices $\mathbf{S}_{a}$ and $\mathbf{S}_{y}$ are quite important in guiding the 1DVAR retrieval to a solution, and determining the right values for $\mathbf{S}_{a}$ and $\mathbf{S}_{y}$ can be a delicate task. The elements of $\mathbf{S}_{a}$ help determine how much leeway is allowed when finding a solution for the state vector: if the assumed errors are small, the state vector will be forced to be more similar to the a priori state vector. The $\mathbf{S}_{y}$ matrix determines how much weight each channel is given in the inversion. Channels whose errors are assumed to be smaller are given more weight, with the simulated $T_{b} \mathrm{~s}$ at these channels forced to match observations more closely.

The assumed a priori error covariances making up $\mathbf{S}_{a}$ are determined by calculating the variances in each state vector parameter from ERA5 reanalysis data (see DK16 for details). For the observations/forward model error covariance matrix $\mathbf{S}_{y}$, many possible sources of error should be considered. We separate these error sources into two categories: those from the radiometer itself and those from the forward model. Both are included in our calculation of $\mathbf{S}_{y}$, though we deem the forward model uncertainties to be greater than the instrument uncertainties.

Potential sources of error in the simulated $T_{b}$ s computed by the forward model include absorption model errors, emissivity model errors, errors in the fixed atmospheric parameters assumed in the forward model (wind speed, sea surface temperature, atmospheric temperature profile, etc.), the simplified representation of the atmosphere as 16 homogeneous levels, and the inability of three PCs to represent the full range of possible water vapor profiles. In cloudy conditions, there are even more sources of uncertainty to consider, such as the vertical distribution of liquid and frozen hydrometeors, their sizes, and their shapes.

While it is possible to estimate the relative contribution to the overall $T_{b}$ uncertainty from any one of the sources listed above, they are not independent of each other. Since variances should only be added to each other if the underlying variables are statistically independent, the channel uncertainties related to forward model errors are estimated as a whole, by comparing simulated $T_{b}$ s from a more detailed representation of the atmosphere to those calculated using the simplified representation of the forward model, with small perturbations to the ancillary parameters.

First, simulated $T_{b} \mathrm{~s}$ are calculated from full ERA5 atmospheric profiles at their native resolution, for oceanic, nonprecipitating profiles that match up with $\mathrm{MetOp}-\mathrm{A}$ overpasses from January 2013. These are compared to a second set of simulated $T_{b} \mathrm{~s}$, again from ERA5, but simplified according to forward model assumptions. That is, the vertical resolution is reduced to 16 pressure layers, the water vapor profile is replaced by the best-fit profile that can be achieved using only three PCs, and the cloud water and ice are redistributed into fixed vertical levels as described in section $3 \mathrm{~b}$. To account for uncertainties in ancillary parameters, small numbers randomly sampled from a Gaussian distribution are added to the parameters 
of SST, salinity, wind speed, wind direction, and layer temperature for each simplified atmospheric profile. The standard deviations of these distributions are $1.0 \mathrm{~K}$ for SST, 0.5 psu for salinity, $2 \mathrm{~m} \mathrm{~s}^{-1}$ for wind speed, $20^{\circ}$ for wind direction, and $2 \mathrm{~K}$ for temperature. Around a hundred million points are forward modeled using both sets of assumptions.

The difference between these two sets of $T_{b}$ s serves as the basis for estimating $\mathbf{S}_{y}$, with the covariances of the simulated minus simulated $T_{b}$ differences making up the elements of the matrix. As for the second component of $\mathbf{S}_{y}$, measurement uncertainties, the published noise equivalent differential temperature (NEDT) values for each radiometer channel are added to the diagonal elements of the matrix to account for radiometric noise. While this procedure of estimating $\mathbf{S}_{y}$ is designed to account for what we believe to be many of the most significant sources of forward model error, particularly for clear-sky conditions, we note that there are other forward model uncertainties that have not been explicitly accounted for. This includes absorption model, emissivity model, and FOV inhomogeneity errors. On the instrument side, there are also uncertainties that are not explicitly accounted for such as errors in the measurement of the temperature of the calibration load, errors from the intrusion of microwave radiation from the spacecraft itself, and errors due to drifts in the local oscillator frequency. While the random components of these errors are not included in the $\mathbf{S}_{y}$ matrix, we do apply radiometric bias corrections, or offsets, to the forward modeled $T_{b} \mathrm{~s}$. These offsets are designed to take into account forward model biases as well as any systematic biases in the measurements. This is explained further below.

\section{1) CONTRIBUTIONS TO FORWARD MODEL UNCERTAINTIES}

A series of experiments were run to attempt to quantify the relative contributions of the various sources of forward model uncertainty to the total uncertainty. In these experiments, a collection of atmospheric profiles, taken from ERA5 and corresponding to a day's worth of observations from $\mathrm{MetO}$ - $A$, were used to repeatedly calculate simulated MHS $T_{b}$ s. In the control experiment, $T_{b}$ s were simulated at native ERA5 resolution, with no simplifying assumptions made. In succeeding experiments, forward model assumptions were incorporated one at time, to test the effect of each on simulated $T_{b} \mathrm{~s}$. First all cloud ice was made to reside between 300 and $400 \mathrm{hPa}$, then all cloud water was put between 800 and $925 \mathrm{hPa}$, then the full vertical profiles of temperature and water vapor were reduced to 16 pressure levels, and then the ERA5 water vapor profile was replaced with the best-fit profile that could be obtained from three
PCs. Finally, the surface wind speeds, wind directions, atmospheric temperature profiles, sea surface salinities, and sea surface temperatures were perturbed as described in section $3 \mathrm{c}$, one at a time.

Figures 1 and 2 show the resulting root-mean-square (RMS) and bias errors, calculated by comparing the simulated $T_{b}$ s from each experiment to the simulated $T_{b}$ s from the previous experiment. The errors are also subset by EIA. Overall, the largest sources of forward model error are the principal component representation of the water vapor profile, the fixed ice cloud level, the fixed liquid cloud level (at 89 and $157 \mathrm{GHz}$ ), and the interpolation down to 16 levels. The interpolation errors are much larger at the $183-\mathrm{GHz}$ channels, which are sensitive to the upper atmosphere where the fixed pressure levels of the forward model are coarser. While the errors from the assumption of a fixed liquid cloud level can be significant (with RMS errors approaching $2 \mathrm{~K}$ at 89 and $157 \mathrm{GHz}$ ), this assumption does not strongly bias the average simulated $T_{b} \mathrm{~s}$, nor is there that large a difference between errors near nadir and errors near the edge of the MHS swath. This is not necessarily the case for other error sources, however. For example, RMS errors stemming from the placement of the ice cloud level is larger at all channels for high EIA compared to low EIA, and this assumption leads to a significantly negative $T_{b}$ bias at most channels in the case of a high EIA, but nearly no bias near nadir. Another example of differing error magnitudes at different view angles can be seen in the case of wind speed. Uncertainties in the surface wind speed only lead to errors in $89-$ and $157-\mathrm{GHz} T_{b} \mathrm{~s}$, as these are the only MHS channels with any sensitivity to the surface. There is more surface sensitivity at low view angles compared to high ones, since the path through the atmosphere is shorter, and thus the RMS errors related to wind speed are greater near nadir than they are at high EIA values.

The bias errors shown in Fig. 2 also include a "residual" category. This value is obtained by calculating the mean difference between the $T_{b}$ s simulated from the full ERA5 profiles (i.e., the control experiment) and the actual observed MHS $T_{b}$ s. One can see that this residual category has a strong view angle dependence, and is most significant at the 89 - and $157-\mathrm{GHz}$ channels. This residual error could theoretically come from a variety of sources. For example, if the ERA5 atmosphere was systematically moister or cloudier than the true atmosphere, one might expect this to bias the simulated $T_{b}$ s. While this cannot be ruled out as a possibility, the fact that this residual bias is fairly consistent across different orbits suggests that this is not the most important contributor. That is to say, if differing meteorology were the primary cause of this $T_{b}$ bias, we would have expected to see a greater degree of variability from orbit to orbit, as different regions of the globe are sampled. This explanation also does not easily explain why the 


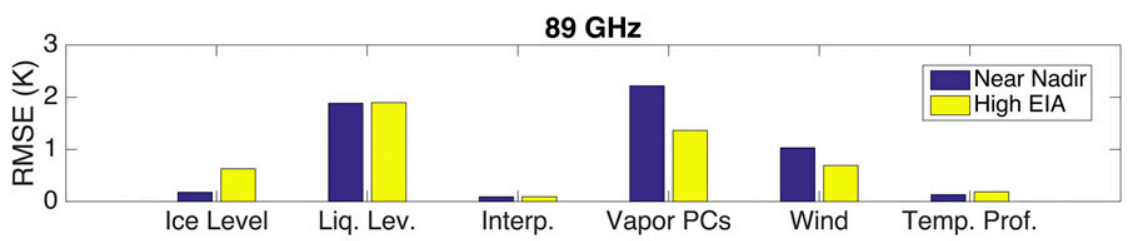

$157 \mathrm{GHz}$

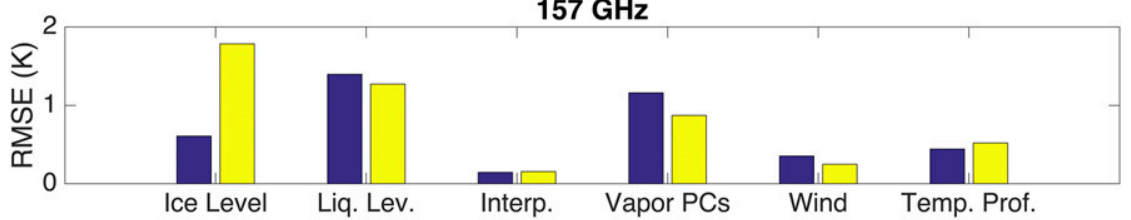

$183+/ 1 \mathrm{GHz}$

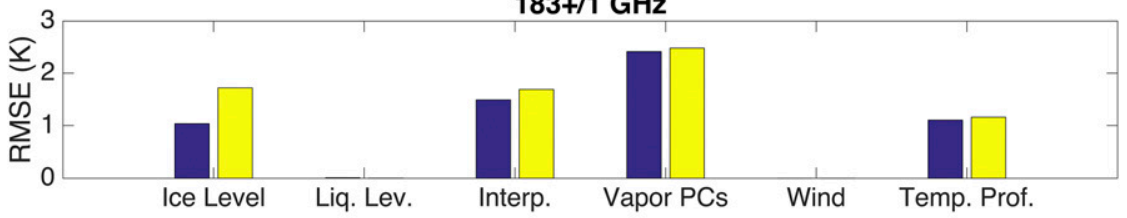

$183+/-3 \mathrm{GHz}$

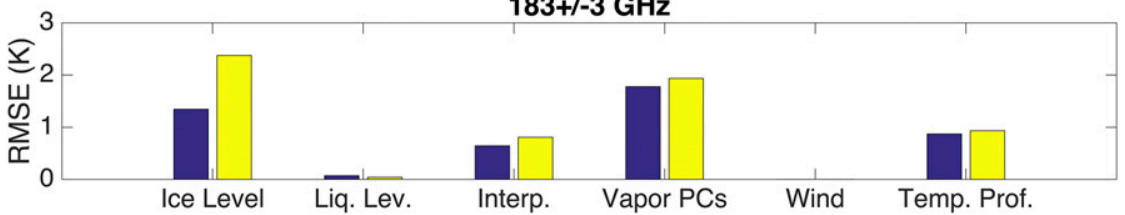

$190 \mathrm{GHz}$

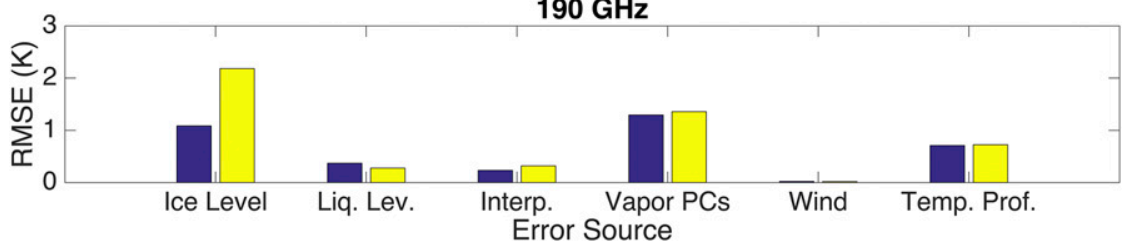

FIG. 1. RMS errors induced at each MHS channel by various forward model assumptions and uncertainties, detailed in the text. The errors related to surface wind direction, sea surface salinity, and SST were found to be quite small, and thus they are not included in the figure. The $T_{b}$ errors from simulated MHS observations taken within $10^{\circ}$ of nadir are in blue, while the yellow bars include only those simulated observations with an EIA of $45^{\circ}$ or greater.

residual bias is greater near the edge of scan than near nadir. Approximations in the absorption model likely account for part of the difference between observed and simulated $T_{b} \mathrm{~s}$, but MonoRTM is believed to be largely unbiased (Clough et al. 2005) and thus the absorption model contribution to the overall bias is probably rather small. Another possibility is that the simulated $T_{b} \mathrm{~s}$ are being biased by an inadequate representation of the size and shape of ice particles. However, if this were the dominant factor, one would not expect to see so much difference between the residual biases at the $183-190-\mathrm{GHz}$ channels, since the scattering effects of ice at these channels should be rather similar. A fourth possibility is that the MHS radiometer itself is biased, with perhaps some sort of $T_{b}$ contamination near the edge of scan that might explain the view angle dependency (John et al. 2013). However, the "observed" $T_{b}$ s we use already have offsets applied to them by the GPM XCAL team (Berg et al. 2016), so any blatant radiometer biases should be accounted for in the intercalibration process. A fifth possible explanation is that the biases are a result of errors in the sea surface emissivity model. This is consistent with the fact that the largest errors are present in the channels with the most sensitivity to the surface. Also, since emissivity is dependent on EIA, this could partly explain the difference in residual biases between high and low values of EIA. A finally possibility is that FOV inhomogeneities are to blame. The generally larger residual biases at high EIA would make some sense, in this context, since the FOV is larger and thus more likely to be inhomogeneous.

In reality, the residual bias likely is a combination of the above factors, and might include other factors, as well. It is worth noting, however, that almost all of these explanations represent a type of forward model bias (the only exception would be if the residual bias were driven solely by a water vapor or cloud coverage bias in ERA5). 

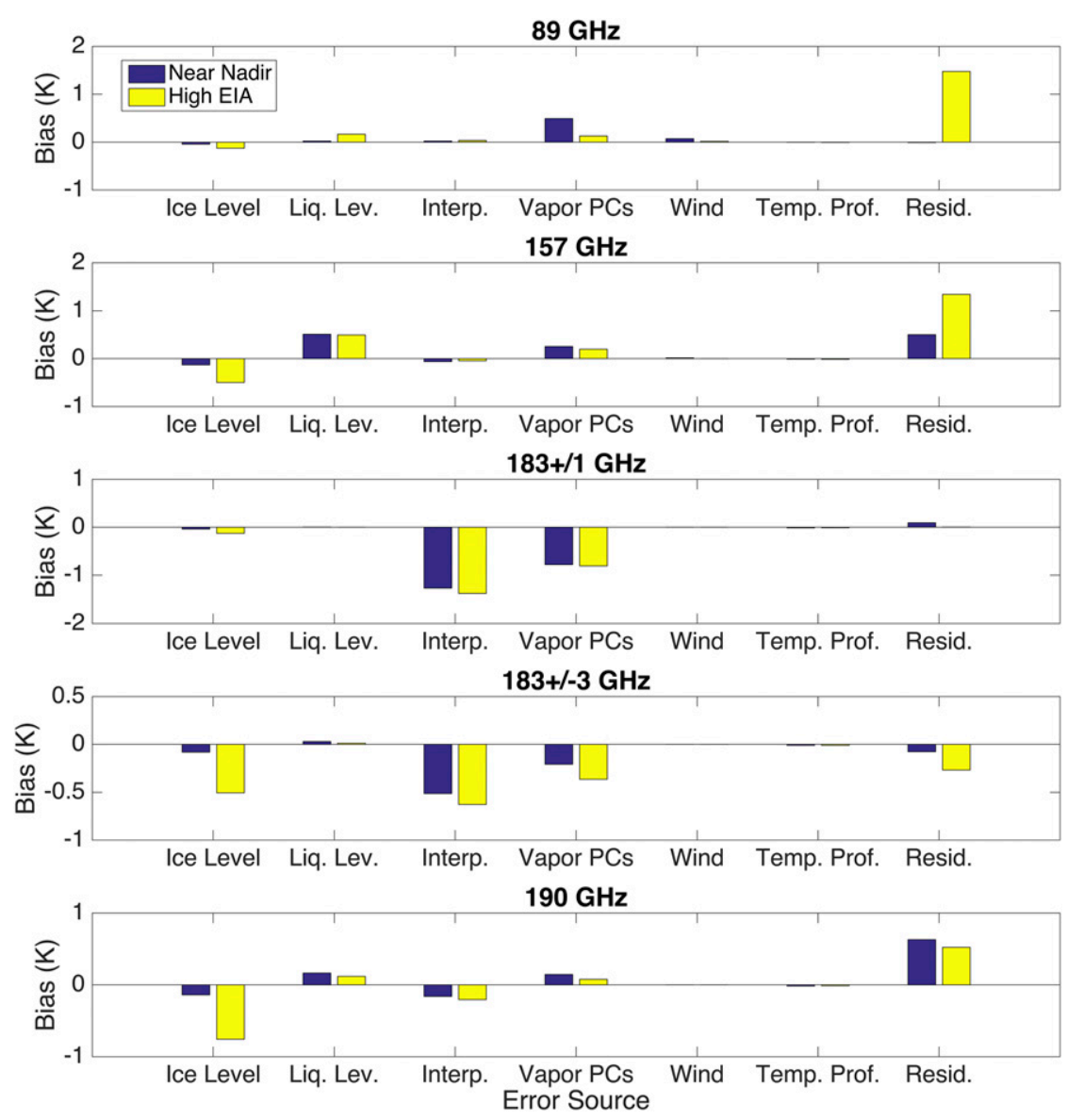

FIG. 2. As in Fig. 1, but for bias errors. The additional "resid." category refers to the mean difference between $T_{b} \mathrm{~s}$ simulated at full ERA5 resolution and those observed by MHS.

Thus, whatever the cause, it is necessary to account for this model bias in order to minimize systematic errors in retrieved variables. We attempt to correct for this forward model bias by applying a corresponding offset, binned by SST and EIA, to the XCAL MHS $T_{b}$ s prior to running the retrieval algorithm. In a fashion similar to the calculation of $\mathbf{S}_{y}$, the offsets are calculated by comparing L1C MetOp-A $T_{b}$ s from January 2013 to simulated $T_{b}$ s created using ERA5 atmospheric profiles, with the simplified vapor and cloud representations of the forward model. The same offsets are applied to all MHS instruments, regardless of satellite. The MiRS algorithm also incorporates bias corrections, but separates offsets only by satellite and scan position, and not by SST or any other variable that could be considered a proxy for the atmospheric state (Boukabara et al. 2011).

\section{2) EARTH INCIDENCE ANGLE AND SST DEPENDENCE OF ERROR ASSUMPTIONS}

As is clear from Figs. 1 and 2, changing the EIA changes the nature of the forward model uncertainties.
This is due to several factors, such as the pathlength through the atmosphere (and thus the sensitivity to various height levels) changing, the size of the instrument footprint changing, and uncertainties in assumed parameters (such as the sea surface emissivity) that are themselves dependent on EIA. Our tests also found that the forward model uncertainties depend strongly on meteorological regime. Generally speaking, forward model uncertainties are largest in high SST regions near the tropics, where there is more water vapor present to absorb upwelling microwave radiation. In particular, tropopause heights are generally higher. Thus, in this regime it is more common to observe significant concentrations of water vapor in the upper atmosphere (above $400 \mathrm{hPa}$ or so), where the vertical resolution of the forward model is coarse, and where the three leading water vapor PCs are able to capture only a narrow range of the true water vapor variability.

DK16 accounted for changes in forward model uncertainty that varied with SST (as a proxy for meteorological regime), but, since they dealt only with conically 
scanning instruments, did not address EIA-related changes. The MiRS algorithm includes bias corrections that depend on scan position, but the modeling error matrix does not have any dependence on EIA, and neither the error matrix nor the bias corrections consider SST. In contrast, we contend that a proper evaluation of the way forward model uncertainties change with EIA and meteorological regime can improve the quality of OE-based retrieval algorithms, and in particular can improve the consistency of retrieved TPW across the full range of view angles for cross-trackscanning microwave instruments. Thus, in our calculation of $\mathbf{S}_{y}$ and of the forward model offsets that we apply to observed $T_{b} \mathrm{~s}$, comparisons are binned both by SST (in 1 -K increments) and by EIA (in $4^{\circ}$ increments). Figure 3 shows four sample $\mathbf{S}_{y}$ matrices, along with the corresponding $T_{b}$ offsets. Note that the magnitude of uncertainty is much greater at 300 than at $275 \mathrm{~K}$, particularly at the highest three MHS frequencies. The bias correction offsets are also much larger. Comparing errors at the same SST but at different EIAs, the general trend is that, for large EIA, channel covariances are slightly reduced at 89 and $157 \mathrm{GHz}$. This is likely due to the reduced surface sensitivity. On the other hand, channel covariances at the other channels are slightly increased, perhaps due to the increased FOV size. The bias correction offsets also have an EIA dependence, with the biases being more negative, in most cases, at high view angles. To make sure there are no major discontinuities in retrieved values from one pixel to a neighboring pixel, both the covariances and the offsets are smoothed using boxcar averaging, and the $T_{b}$ offsets that are applied to any given pixel are calculated from a bilinear interpolation of the EIA/SST binned offsets.

\section{d. Matchups with SuomiNet TPW estimates}

To compare retrieved TPW with GPS-based estimates from SuomiNet, we identified MHS observations which came no more than $15 \mathrm{~min}$ before or after a SuomiNet estimate and which were centered on a location no greater than $0.5^{\circ}$ away in latitude or longitude. MHS pixels were only eligible for comparison if both the MiRS and CSU 1DVAR algorithms reached convergence. When comparing SuomiNet TPW values to MERRA-2, the MERRA-2 oceanic grid point closest to the GPS station was used.

The CSU 1DVAR algorithm only converges over the ocean, so the only GPS stations that produce observations meeting the matchup requirements outlined above are stations located on small islands and some coastal stations. Figure 4 shows the location of the 45 GPS stations that yielded at least $10 \mathrm{TPW}$ estimates coincident with a MetOp-B MHS overpass during the month of June 2016.
Note that, while most of the GPS stations are located at a low elevation, they are all located above sea level, and measure only the precipitable water in the atmosphere above that elevation. Thus, one would expect the SuomiNet estimate of TPW to be biased low relative to the TPW of the surrounding oceanic areas. To allow for a direct comparison with the sea level-based TPW estimates from MiRS, CSU 1DVAR, and MERRA-2, we use a simple model to account for the water vapor in the atmospheric column above the ocean surface but below the station elevation. Following the example of Mears et al. (2015), we assume the intervening atmosphere has a temperature equal to the SST used in the IDVAR retrieval, with a relative humidity of $80 \%$. Since nearly all of the GPS stations considered lie at an elevation below $100 \mathrm{~m}$ above sea level, the adjustments resulting from this procedure are generally small (typically around $1 \mathrm{~mm}$ ). The exceptions are the stations on Mauritius Island (elevation: $427 \mathrm{~m}$ ) and Christmas Island (elevation: $266 \mathrm{~m}$ ). However, even for these stations, the simple adjustment technique seems to be satisfactory, with error statistics that are consistent with the lower-elevation GPS stations.

\section{Results}

\section{a. Comparison of CSU 1DVAR and MiRS algorithms}

In the results that follow we use MiRS output as one of the main reference points by which to judge the performance of the CSU 1DVAR algorithm. We have used MiRS as a source of comparison because it is a widely used, state-of-the-art, flexible retrieval algorithm that is underpinned by much of the same mathematics as the CSU 1DVAR algorithm. Given that both algorithms are built on the OE framework, it is helpful to consider the differences between the two algorithms, so as to better understand the potential reasons why the two algorithms might yield different results. Table 1 outlines what we believe to be some of the most important differences.

One key difference is that the algorithms use different radiometric inputs. As mentioned earlier, CSU 1DVAR does not take into account AMSU-A channels. For the results presented here, however, what is probably more significant is that the MiRS algorithm uses operational level-1B (L1B) brightness temperatures, while the CSU 1DVAR uses L1C intercalibrated $T_{b} \mathrm{~s}$. This means that the radiometric bias corrections computed by MiRS incorporate both forward model biases (which should in principle be very similar from one satellite to another) as well as satellite-specific calibration differences. The use of intercalibrated $T_{b}$ s by CSU 1DVAR allows us to compute a single set of offsets that can be applied to all satellites carrying a given instrument. The resulting 


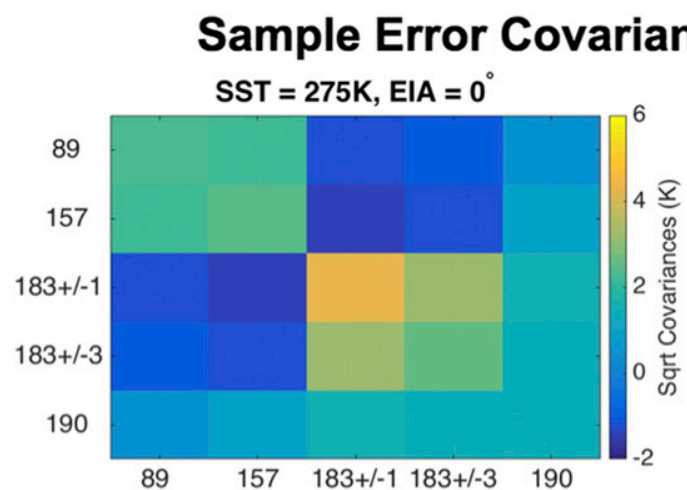

\section{ce Matrices and Offsets}
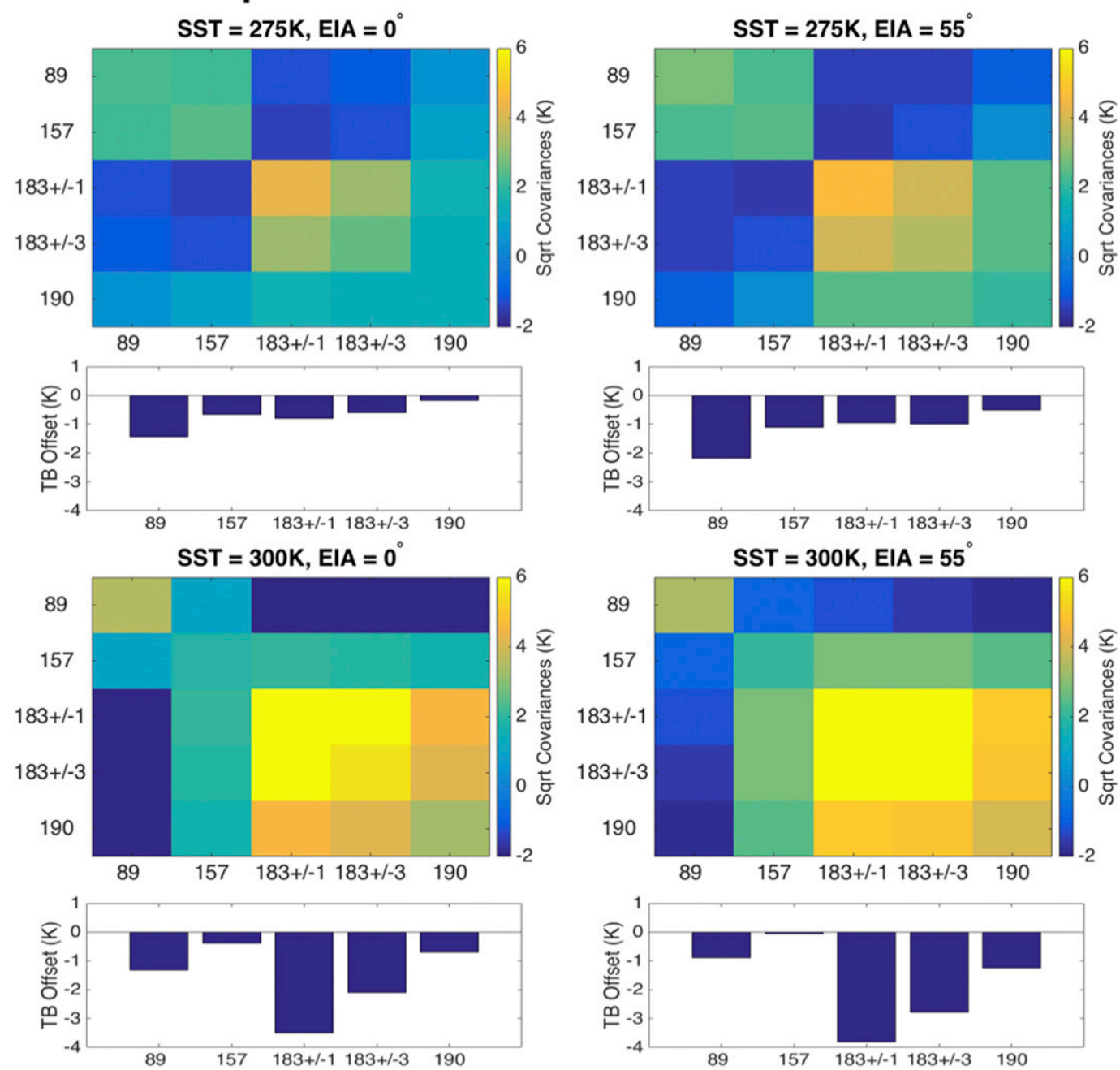

FIG. 3. Sample forward model error covariance matrices, for the SST bins 275-276 and 300-301 K, and EIA bins $0^{\circ}-4^{\circ}$ and $52^{\circ}-56^{\circ}$. Values are given as the square root of the covariances so as to have units of kelvins. Negative covariances are shown as -1 times the square root of the absolute value of the covariance. Below each covariance matrix is the associated forward model bias correction at each channel for the given SST/EIA combination. This represents the average difference between $T_{b}$ s simulated from the simplified forward model and those observed by the MHS instrument on MetOp-A.

offsets thus represent mostly forward models biases as opposed to calibration errors.

Another important difference is that the CSU 1DVAR algorithm incorporates a priori information about the water vapor profile from ERA5, while MiRS relies only upon climatological restraints. While the CSU 1DVAR retrieved TPW is not overly sensitive to the choice of a priori, it does have nontrivial influence on the final answer. Thus, when evaluating the performance of the two algorithms, one should keep in mind the additional information available to CSU 1DVAR through the a priori. The dependence upon only climatological constraints by MiRS allows for retrieved products to be available in near-real time, which is not currently possible with CSU 1DVAR. It should also be noted that MHS data are assimilated into the ERA5 reanalysis, so there is a subtle influence on the a priori by the observations. However, we believe this is not a major cause for concern. In an experiment in which GFS 6-h forecasts were used to provide a priori water profiles instead of ERA5, retrieved TPW tended to slightly increase, but there was little effect on either the bulk error statistics or the pattern of TPW biases across the scan. Crucially, as we show in section $4 \mathrm{e}$, the reduction in EIA-related TPW biases is largely insensitive to the choice of a priori, and this is the area in which we feel the CSU 1DVAR 


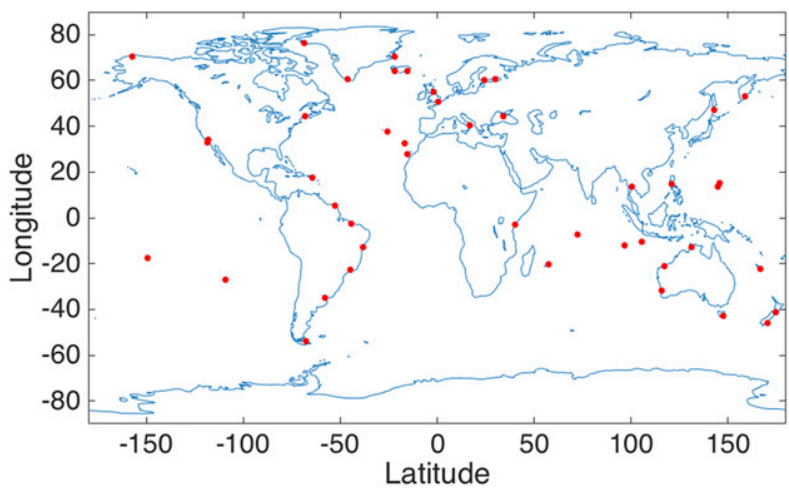

FIG. 4. Location of the SuomiNet coastal and island GPS stations used to validate oceanic TPW estimates.

represents an advancement compared to other optimal estimation algorithms.

\section{b. Qualitative comparison of retrieved fields}

The CSU 1DVAR produces retrieved values that are broadly consistent with those from MiRS and MERRA2 reanalysis. Figure 5 shows a sample $\mathrm{MetO} P$ - $B$ scene as retrieved by MiRS and the CSU 1DVAR, along with corresponding values taken from the ERA5 (whose water vapor profiles, though not LWP or IWP fields, are used in the 1DVAR a priori state vector) and MERRA-2. All products show TPW fields that are rather similar to one another. They also put cloud water in roughly the same place, though there are some significant differences. MiRS and CSU 1DVAR agree with each other in terms of LWP much more closely than either of the reanalyses agree with each other or with the retrieved products. MERRA-2, in particular, has generally larger LWP values, though it has less in the cloud cluster near the top of the image, where the other products agree the largest LWP values reside. CSU 1DVAR, ERA5, and MERRA-2 also put frozen hydrometeors in roughly the same place, though ERA5 has generally larger IWP values than MERRA-2, and the CSU 1DVAR gives still larger values. The differences are likely related to differences in the way ice particle size distributions are parameterized. On the other hand, MiRS IWP values [technically graupel water path (GWP) in the MiRS algorithm] are much lower. We note that newer versions of the MiRS algorithm (version 11.0 and higher) use an updated radiative transfer model and generally retrieve much higher amounts of graupel water.

\section{c. Statistical comparison with MiRS}

Figure 6 shows density plots of retrieved values of TPW and LWP from MiRS versus those from CSU 1DVAR, for the MetOp-B satellite during the month of June 2016. MetOp-B is used here because it is the only satellite carrying MHS for which MiRS orbital data files were available at full MHS resolution in 2016, as opposed to the more coarse resolution of AMSU-A. This allows for a more direct comparison with CSU 1DVAR; however, the results are very similar when considering retrievals from MetOp-A, NOAA-18, or NOAA-19, or retrievals made during other months. Retrieved TPW values are in very good overall agreement, with a correlation coefficient of 0.985 and a root-mean-square difference of $2.75 \mathrm{~mm}$. On average, CSU 1DVAR TPW is biased $-0.60 \mathrm{~mm}$ lower than MiRS TPW. TPW values are biased slightly high relative to MiRS in dry atmospheres and slightly low in moist ones. The relationship between CSU 1DVAR and MiRS LWP is noisier, but the correlation coefficient of 0.62 is higher than the correlation between MiRS and either MERRA-2 $(r=0.30)$ or ERA5 $(r=0.51)$. On average, retrieved LWP is biased slightly low relative to MiRS (by $7.2 \mathrm{~g} \mathrm{~m}^{-2}$ ). The highdensity strips along both axes in the bottom panel of Fig. 6 indicate that there are a number of pixels for which CSU IDVAR retrieves significant cloud water while MiRS does not, and vice versa. An examination of individual retrieved orbits does not reveal any obvious patterns as to when these disagreements occur, but the discrepancies could be related to the use of AMSU-A 23.8- and

TABLE 1. Summary of key differences between the MiRS and CSU 1DVAR algorithms.

\begin{tabular}{|c|c|c|}
\hline & CSU 1DVAR & MiRS \\
\hline Input level-1 data & XCAL L1C $T_{b} \mathrm{~s}$ & Operational L1B data \\
\hline Channels used & MHS only & AMSU-A and MHS \\
\hline Retrieved variables & Water vapor (profile), LWP, IWP & $\begin{array}{l}\text { Temperature (profile), WV (profile), } \\
\text { surface emissivity, skin temperature, } \\
\text { LWP (profile), GWP (profile) }\end{array}$ \\
\hline Radiometric bias correction & $\begin{array}{l}\text { Function of EIA and SST; same } \\
\text { offsets applied to all satellites }\end{array}$ & $\begin{array}{l}\text { Function of EIA only but computed } \\
\text { separately for each satellite }\end{array}$ \\
\hline Forward model error covariance matrix $\mathbf{S}_{y}$ & Function of EIA and SST & Fixed, diagonal matrix \\
\hline A priori constraint & $\begin{array}{l}\text { ERA5 for temperature, water vapor, } \\
\text { wind speed, SST, and salinity }\end{array}$ & $\begin{array}{l}\text { Climatology with dependence on } \\
\text { location, month, time of day }\end{array}$ \\
\hline Absorption model & MonoRTM & CRTM \\
\hline
\end{tabular}



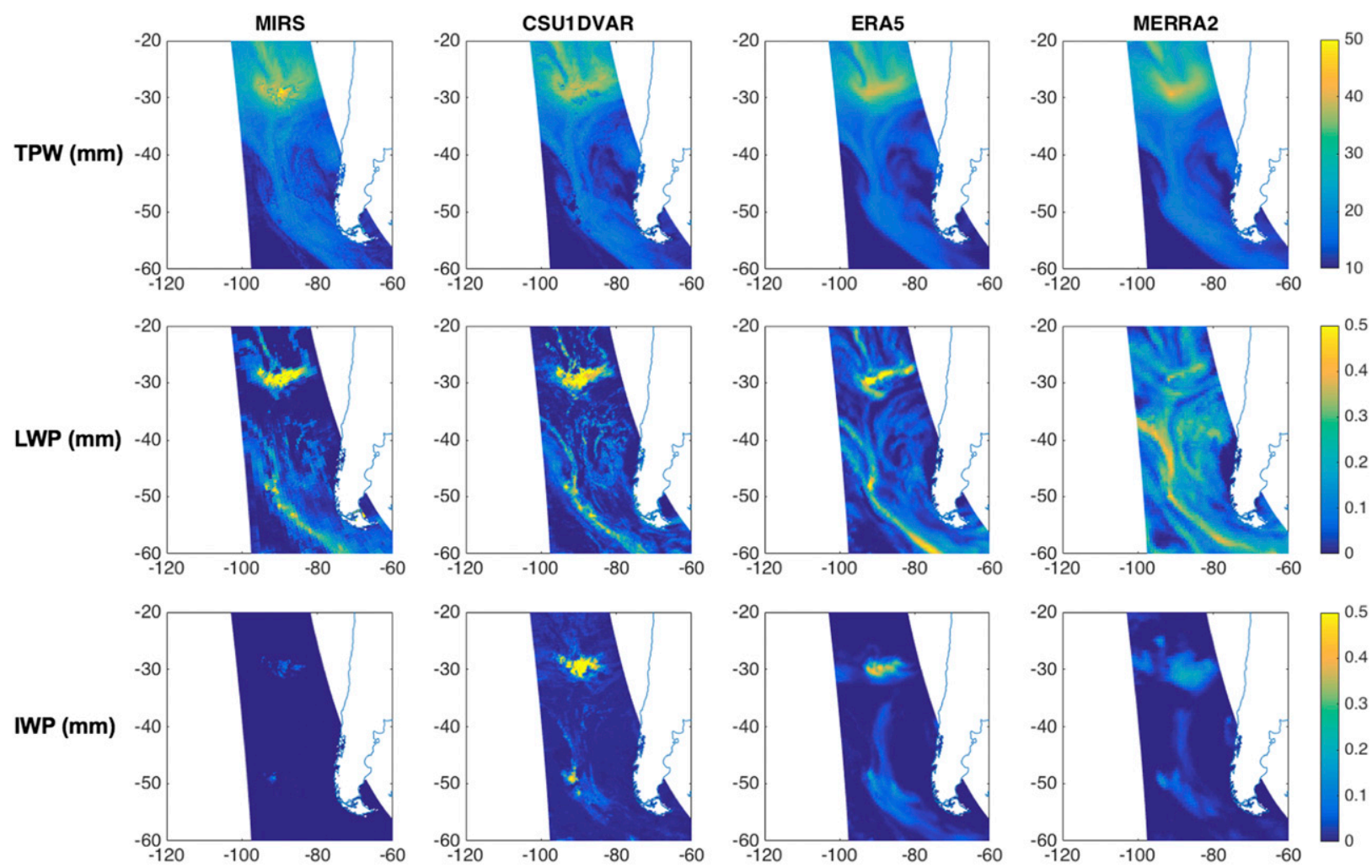

FIG. 5. TPW, LWP, and IWP from MiRS, CSU 1DVAR, ERA5, and MERRA-2, for a MetOp-B overpass off the western coast of South America on 1 Jun 2016. For MiRS, GWP is plotted in place of IWP.

31.4-GHz channels by MiRS, which in theory should give additional sensitivity to LWP.

\section{d. TPW validation against GPS ground-based measurements}

A second quantitative assessment of the CSU 1DVAR water vapor retrieval was conducted by comparing retrieved TPW from all satellites carrying MHS to near-coincident estimates from SuomiNet GPS stations, following the procedure described in section 3d. Figure 7 compares June 2016 SuomiNet (altitude adjusted) TPW estimates to TPW from CSU 1DVAR, MiRS, and MERRA-2, for each satellite and as a function of SuomiNet TPW. Summary statistics from all matchups during the year 2016 are given in Table 2. Our findings suggest that oceanic TPW from MiRS is biased high, similar to findings by Boukabara et al. (2010) that MiRS TPW from $\mathrm{MetOp}$ - $A$ and $N O A A-18$ is biased high relative to radiosondes and assimilation system analyses. This high bias appears to be more of an issue in low-TPW environments. CSU 1DVAR, on the other hand, is biased high relative to the other products in moist environments. Bias and RMS errors are generally lower for CSU 1DVAR than for MiRS, with a slightly better correlation coefficient, though neither is significantly better than reanalysis.
CSU 1DVAR biases are also more consistent from one MHS satellites to another than are MiRS biases, which is likely related to the use of L1C intercalibrated $T_{b} \mathrm{~s}$ as opposed to the L1B product used by MiRS. Overall, it appears that the CSU 1DVAR retrieval of MHS TPW performs at least as well as if not better than MiRS, despite the fact that the algorithm makes no direct use of the simultaneous observations by AMSU-A. However, it should be noted that the algorithm takes into account ancillary information from reanalysis that MiRS does not.

\section{e. TPW biases related to EIA}

We believe the main strength of the CSU 1DVAR algorithm is its resistance to view angle-related biases, due to the fact that assumed forward model biases and uncertainties are allowed to change with EIA. Figure 8 shows the average bias of MiRS and CSU 1DVAR retrieved TPW values relative to MERRA-2, as a function of EIA. All satellites demonstrate a remarkably similar pattern with respect to the MiRS biases, with MiRS TPW tending to be $1-2 \mathrm{~mm}$ higher than MERRA-2 at most view angles, but with a rapidly decreasing bias at EIAs greater than approximately $40^{\circ}$. However, when the CSU 1DVAR algorithm is run with assumed errors that change with SST and view angle, the retrieved TPW 

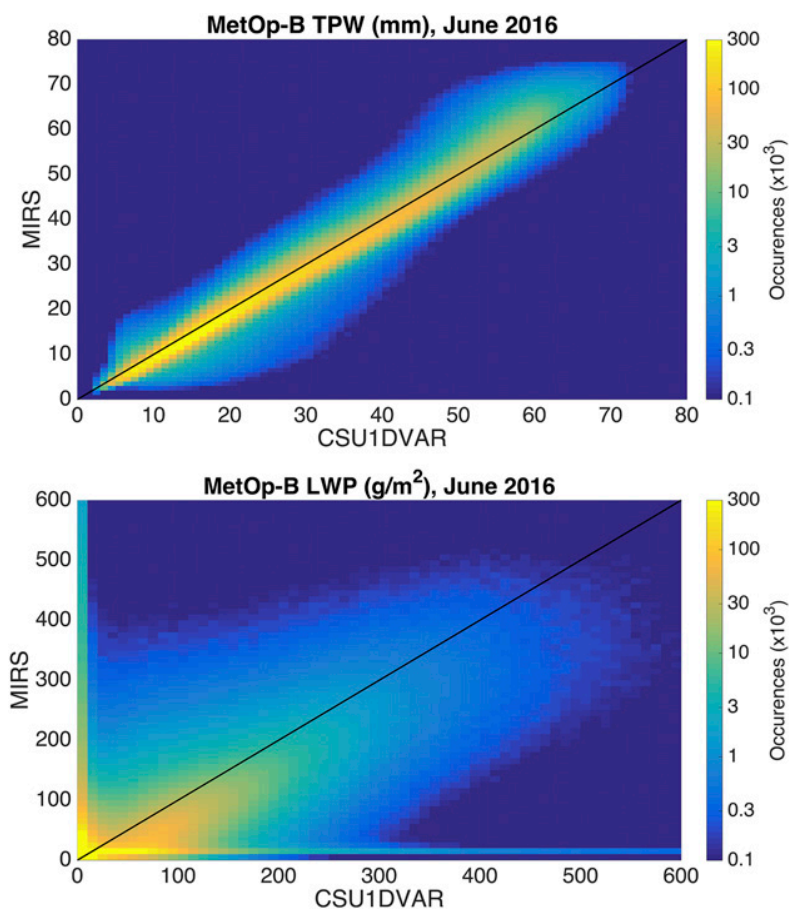

FIG. 6. Density plots of retrieved (top) TPW and (bottom) LWP against corresponding retrieved values from MiRS, for all oceanic $M e t O p-B$ pixels from the month of June 2016 for which both algorithms converged to a solution. The black line in each plot is the 1:1 line.

is not only in better agreement with MERRA-2, but is also almost entirely free from EIA-dependent biases.

Given the many differences between MiRS and CSU IDVAR discussed above, it is natural to ask which factors are most important in reducing the EIAdependent TPW bias. For instance, the ERA5 water vapor profiles used as a priori information do not themselves show a dependency on satellite view angle. If the retrieved TPW were strongly tied to the a priori, then this could explain the lack of EIA-related bias in the retrieved TPW. However, this does not seem to be the case. As shown in Fig. 8, when the CSU 1DVAR retrieval algorithm is run with a constant $\mathbf{S}_{y}$ matrix and constant $\mathrm{T}_{\mathrm{b}}$ offsets, but with the exact same a priori profiles, a pattern similar to the MiRS response is seen in the retrieved TPW. In addition, to test the effect biased a priori information might have on the retrieval, a separate experiment was performed in which output from MiRS was used to provide the a priori water vapor profiles to the CSU 1DVAR algorithm. New retrievals were then run on June 2016 MetOp-B data and the retrieved TPW compared to the original output from CSU 1DVAR ERA5 a priori information. As shown in Fig. 9, this had the effect of slightly increasing the amount of retrieved TPW; however, the EIA-related bias was still mostly eliminated, suggesting that it is not the a priori constraints that are responsible for eliminating scan-dependent bias in the CSU 1DVAR algorithm.

A similar experiment was conducted to test the relative impact of allowing the $\mathbf{S}_{y}$ matrix to change versus allowing the forward model offsets to change according to the EIA and SST. This research was partially motivated by a finding that for synthetic, vapor-only retrievals (not shown), increasing the assumed errors near the edge of scan reduced TPW biases in these areas. However, as shown in Fig. 9, for the full MHS retrieval, when the assumed uncertainties in $\mathbf{S}_{y}$ are already larger to account for the possibilities of clouds, this effect is muted. Changing $\mathbf{S}_{y}$ alone does slightly reduce the EIA-related bias in retrieved TPW (the difference between the nadir and edge-of-scan mean biases decreases by about $0.2 \mathrm{~mm}$ ), but allowing the offsets to change is much more important in reducing the TPW bias.

Last, given that CSU 1DVAR bins offsets and $\mathbf{S}_{y}$ by both EIA and SST, an experiment was conducted to test the effect of considering either factor by itself. Figure 9 shows that both changes result in differences compared to assuming a fixed $\mathbf{S}_{y}$ and fixed offsets. Binning by SST overall increases the mean retrieved TPW; however, the consistent increase shown in Fig. 9 masks the fact that binning by SST serves to generally increase retrieved TPW in the tropics and decrease it elsewhere, resulting in a net increase in the global mean. Binning error assumptions by EIA appears to be more important in reducing the view angle-related bias, but this step alone is not sufficient to eliminate it. The overall offsets used in the CSU 1DVAR algorithm are not linear in either the EIA or SST dimensions, and it is only when both scan position and atmospheric regime are considered together that the algorithm is able to nearly eliminate the EIA-dependent TPW bias.

\section{Conclusions}

The CSU 1DVAR retrieval algorithm is an extremely flexible algorithm for retrieving TPW, LWP, and IWP from virtually any spaceborne passive microwave radiometer. While past studies have applied the algorithm to conically scanning instruments, we have demonstrated that the algorithm can be used with cross-track-scanning instruments as well, by applying the algorithm to MHS-observed radiances. Retrieved LWP values are in general agreement with MiRS, and comparisons of retrieved TPW with estimates from SuomiNet and MERRA-2 suggest that, if anything, 

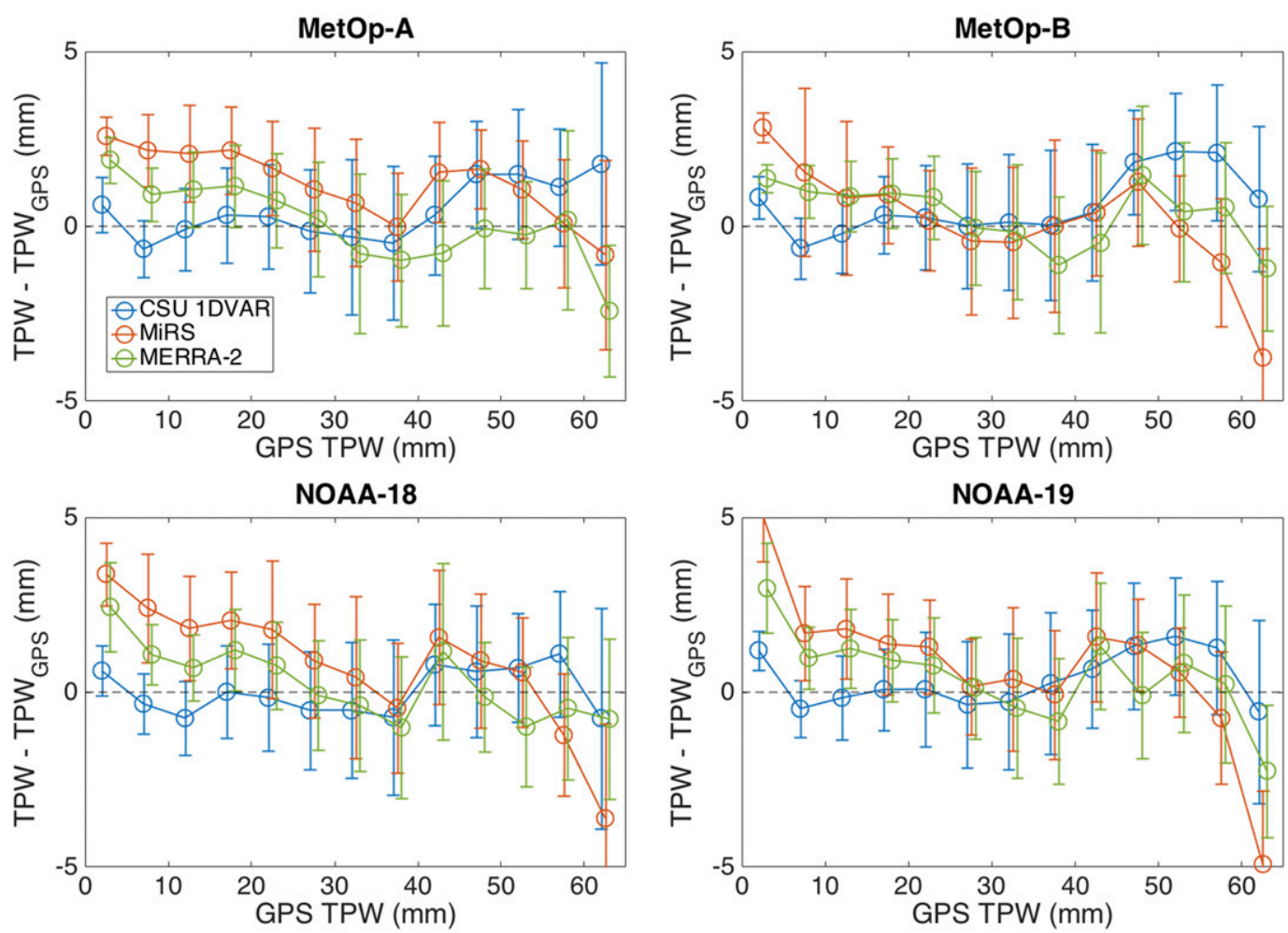

FIG. 7. Mean and standard deviations of the difference between CSU 1DVAR (blue), MiRS (red), and MERRA2 (green) TPW estimates and near-coincident altitude-adjusted estimates from SuomiNet GPS stations, for each satellite carrying an MHS instrument during the month of June 2016. Matchups are binned by 5-mm TPW increments, with the binning determined by the GPS estimate.

CSU 1DVAR estimates of TPW might be more reliable than MiRS.

Our goal in presenting this work is not to suggest that the CSU 1DVAR algorithm is categorically better than MiRS or any other retrieval algorithm, but rather to point to an important way that we believe MiRS and other physically based retrieval algorithms can improve, particularly for cross-track-scanning instruments. We readily acknowledge than MiRS has certain advantages over the CSU 1DVAR algorithm, such as its ability to retrieve values over land, its greater number of retrieved variables, and its nonreliance on any sort of weather model for a priori information. One of the distinctive features of the CSU 1DVAR algorithm is the way that forward model error assumptions are allowed to change based on the SST and EIA of the pixel being considered, and we have demonstrated that this approach nearly eliminates EIA-related biases in the retrieval of TPW from all MHS instruments. Only simulated $T_{b}$ s from MetOp-A (from January 2013) are used when calculating the $\mathbf{S}_{y}$ matrices and associated $T_{b}$ offsets, yet the same error assumptions are able to nearly eliminate the view angle-related TPW bias not only for $\mathrm{Met} O p$ - $A$ but for all other MHS satellites as well, for a completely different time period. This suggests that the errors estimated by the $\mathbf{S}_{y}$ matrix and the radiometric biases

TABLE 2. Bias (mm), RMS error (mm), and correlation coefficient between 2016 SuomiNet altitude-adjusted TPW estimates and near-coincident estimates from MiRS, CSU 1DVAR, and MERRA-2, categorized by MHS satellite.

\begin{tabular}{|c|c|c|c|c|c|c|c|c|c|c|c|c|}
\hline \multirow[b]{2}{*}{ Product } & \multicolumn{3}{|c|}{$M e t O p-A$} & \multicolumn{3}{|c|}{$M e t O p-B$} & \multicolumn{3}{|c|}{$N O A A-18$} & \multicolumn{3}{|c|}{ NOAA-19 } \\
\hline & Bias & RMSE & $\begin{array}{c}\text { Correlation } \\
\text { coef }\end{array}$ & Bias & RMSE & $\begin{array}{l}\text { Correlation } \\
\text { coef }\end{array}$ & Bias & RMSE & $\begin{array}{c}\text { Correlation } \\
\text { coef }\end{array}$ & Bias & RMSE & $\begin{array}{c}\text { Correlation } \\
\text { coef }\end{array}$ \\
\hline MiRS & 1.32 & 3.51 & 0.978 & 0.63 & 4.33 & 0.960 & 1.17 & 4.08 & 0.970 & 0.66 & 3.90 & 0.971 \\
\hline CSU 1DVAR & 0.39 & 3.31 & 0.980 & 0.53 & 3.52 & 0.977 & 0.12 & 3.48 & 0.979 & 0.17 & 3.91 & 0.971 \\
\hline MERRA-2 & 0.20 & 3.28 & 0.978 & 0.21 & 3.45 & 0.975 & 0.14 & 3.13 & 0.981 & 0.10 & 3.73 & 0.972 \\
\hline
\end{tabular}


TPW Compared to MERRA-2, June 2016
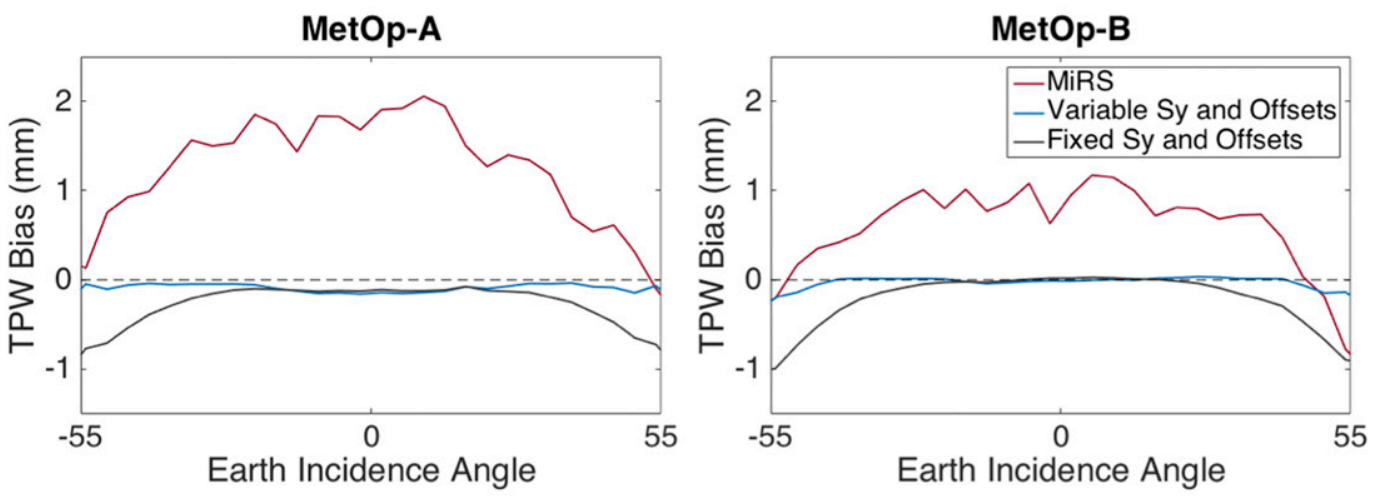

NOAA-18
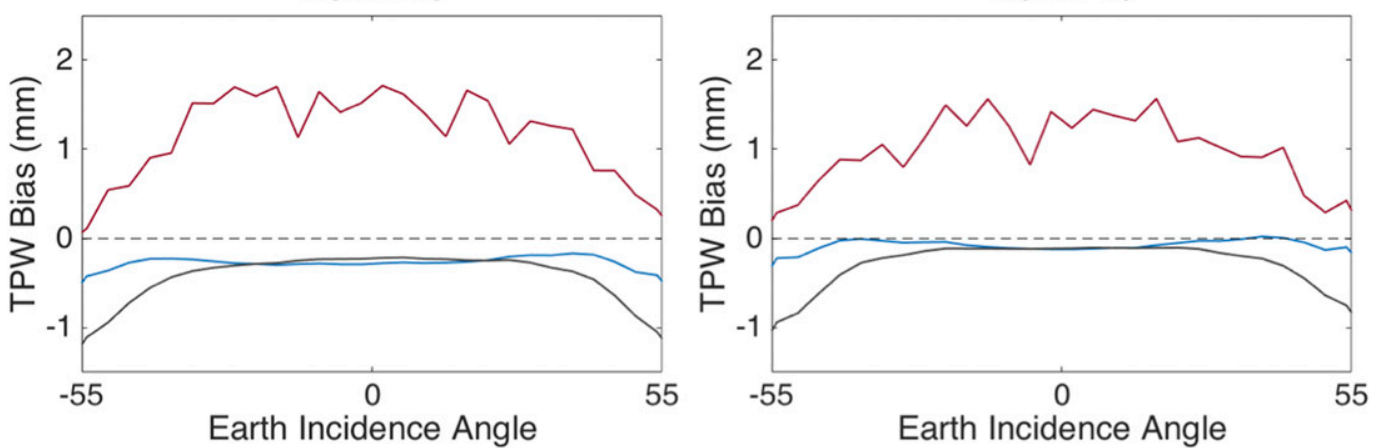

FIG. 8. Average difference between retrieved TPW and MERRA-2 TPW, as a function of EIA and for MHS observations from (top left) $M e t O p-A$, (top right) $M e t O p-B$, (bottom left) $N O A A-18$, and (bottom right) $N O A A-19$. CSU 1DVAR results using fixed error assumptions are shown in blue, 1DVAR results using the variable error assumptions described in the text are shown in gray, and MiRS results are shown in red.

corrections are mostly reflective of forward model errors, rather than instrument errors, and that the XCAL intercalibration team has done a good job of creating consistency between the satellite measurements.

The fact that the pattern of MiRS EIA-related TPW biases is so similar across all MHS satellites is supportive of the idea that the inconsistency across the scan is primarily a result of an algorithm shortcoming, or possibly an error common to all MHS instruments (though this seems less likely), rather than an individual sensor calibration issue. A similar pattern is seen in the CSU 1DVAR-retrieved TPW when constant error assumptions are used, but is eliminated once the forward model error covariance matrix and the associated forward model $T_{b}$ offsets are allowed to grow or shrink according to the SST and EIA. It would be relatively straightforward to apply this approach to other OE-based schemes that rely on assumptions about forward model and observational uncertainties, including MiRS.

We have identified some physical reasons why error assumptions should not be constant. The increased water vapor burden in a warmer atmospheric profile means that MHS channels are sensitive to higher levels of the atmosphere relative to a colder/dryer profile. This increases the uncertainty at channels near the $183-\mathrm{GHz}$ water vapor absorption line, while decreasing the uncertainty at channels such as 89 or $157 \mathrm{GHz}$ for which uncertainties in surface parameters (e.g., emissivity or wind) make up a large part of the total uncertainty in dry atmospheres. To a lesser extent, increasing the EIA has a similar effect, since the increased pathlength means that the channel weighting functions peak slightly higher in the atmosphere and have reduced sensitivity to the surface. However, work remains to be done to better understand residual errors that depend on EIA. While the approach presented here is able to properly account for them and allow for consistent retrievals no matter the EIA, it would be better to be able to pinpoint precisely their cause, whether it be emissivity model errors, FOV inhomogeneities, an instrument design flaw, or something else entirely.

As the focus of satellite meteorology shifts more toward process understanding, and constellations of small satellites become more common, it will be important to be able to consistently compare observations made by one satellite instrument to those made by another, 


\section{Contributions to Scan Position Bias Reduction}
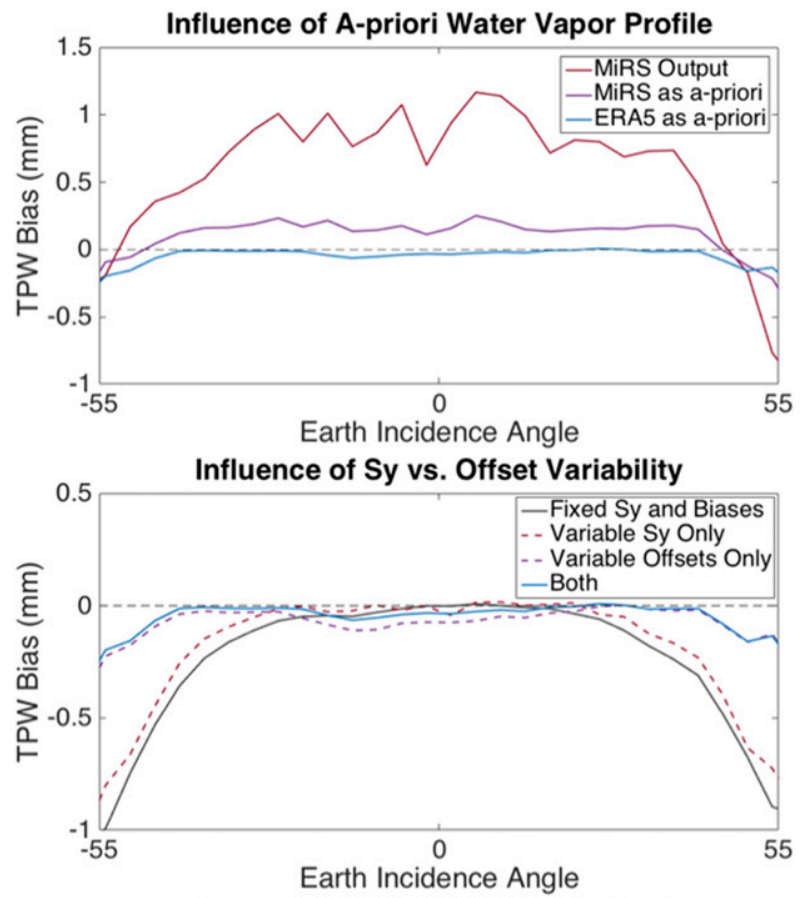

Influence of SST and EIA Error Binning

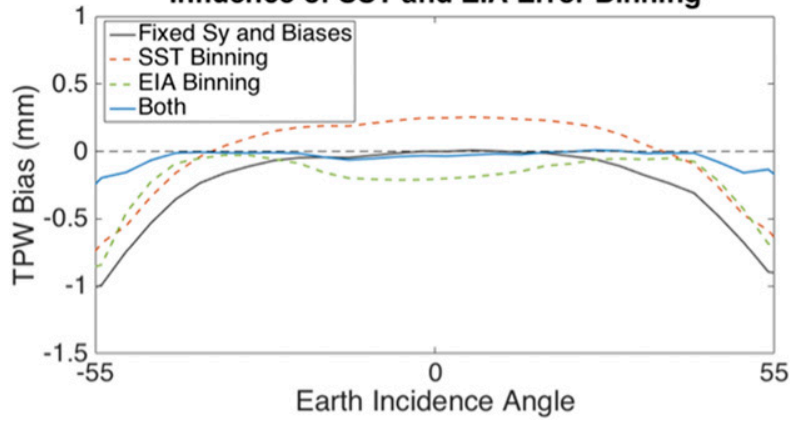

FIG. 9. Average difference between retrieved TPW and MERRA-2 TPW as a function of EIA, for MetOp-B observations from June 2016: (top) Effect of using MiRS-retrieved water vapor profiles as the a priori profile for the CSU 1DVAR retrieval. (middle) Effects of allowing either only $\mathbf{S}_{y}$ or only the forward model offsets to vary based on EIA and SST. (bottom) Effects of stratifying $\mathbf{S}_{y}$ and the forward model offsets by either SST or EIA alone.

possibly viewing the scene from a different angle. The CSU 1DVAR algorithm should be especially well suited to this purpose, given its flexibility for use with a multitude of channel combinations and its explicit handling of view angle-induced uncertainties.

Acknowledgments. The authors thank Drs. David Duncan and Wesley Berg for numerous helpful discussions throughout the development of the CSU 1DVAR algorithm. The work was supported by NASA Earth Venture Program Grant NNX15AP56G.

\section{REFERENCES}

Alishouse, J. C., S. A. Snyder, J. Vongsathorn, and R. R. Ferraro, 1990: Determination of oceanic total precipitable water from the SSM/I. IEEE Trans. Geosci. Remote Sens., 28, 811-816, https://doi.org/10.1109/36.58967.

Bennartz, R., 2007: Global assessment of marine boundary layer cloud droplet number concentration from satellite. J. Geophys. Res., 112, D02201, https://doi.org/10.1029/2006JD007547.

Berg, W., and Coauthors, 2016: Intercalibration of the GPM microwave radiometer constellation. J. Atmos. Oceanic Technol., 33, 2639-2654, https://doi.org/10.1175/JTECH-D-16-0100.1.

Blackwell, W. J., and Coauthors, 2018: An overview of the TROPICS NASA Earth Venture mission. Quart. J. Roy. Meteor. Soc., 144, 16-26, https://doi.org/10.1002/qj.3290.

Boucher, O., and Coauthors, 2013: Clouds and aerosols. Climate Change 2013: The Physical Science Basis, T. F. Stocker et al., Eds., Cambridge University Press, 571-657.

Boukabara, S.-A., K. Garrett, and W. Chen, 2010: Global coverage of total precipitable water using a microwave variational algorithm. IEEE Trans. Geosci. Remote Sens., 48, 3608-3621, https://doi.org/10.1109/TGRS.2010.2048035.

_ _ and Coauthors, 2011: MiRS: An all-weather 1DVAR satellite data assimilation and retrieval system. IEEE Trans. Geosci. Remote Sens., 49, 3249-3272, https://doi.org/10.1109/ TGRS.2011.2158438.

Clough, S. A., M. W. Shephard, E. J. Mlawer, J. S. Delamere, M. J. Iacono, K. Cady-Pereira, S. Boukabara, and P. D. Brown, 2005: Atmospheric radiative transfer modeling: A summary of the AER codes. J. Quant. Spectrosc. Radiat. Transfer, 91, 233-244, https://doi.org/10.1016/j.jqsrt.2004.05.058.

Deblonde, G., and S. English, 2003: One-dimensional variation retrievals from SSMIS-simulated observations. J. Appl. Meteor., 42, 1406-1420, https://doi.org/10.1175/1520-0450(2003) 042<1406:OVRFSO >2.0.CO;2.

Draine, B. T., and P. J. Flatau, 1994: Discrete-dipole approximation for scattering calculations. J. Opt. Soc. Amer., 11A, 14911499, https://doi.org/10.1364/JOSAA.11.001491.

Duncan, D. I., and C. D. Kummerow, 2016: A 1DVAR retrieval applied to GMI: Algorithm description, validation, and sensitivities. J. Geophys. Res. Atmos., 121, 7415-7429, https://doi.org/ 10.1002/2016JD024808.

ECMWF, 2017: ERA5 reanalysis. National Center for Atmospheric Research Computational and Information Systems Laboratory, accessed 1 June 2018, https://doi.org/10.5065/D6X34W69.

Elsaesser, G. S., and C. D. Kummerow, 2008: Toward a fully parametric retrieval of the nonraining parameters over the global oceans. J. Appl. Meteor. Climatol., 47, 1599-1618, https://doi.org/ 10.1175/2007JAMC1712.1.

Field, P. R., A. J. Heymsfield, and A. Bansemer, 2007: Snow size distribution parameterization for midlatitude and tropical ice clouds. J. Atmos. Sci., 64, 4346-4364, https://doi.org/10.1175/ 2007JAS2344.1.

Forsythe, J. M., S. Q. Kidder, K. K. Fuell, A. Leroy, G. J. Jedlovec, and A. S. Jones, 2015: A multisensory, blended, layered water vapor product for weather analysis and forecasting. J. Oper. Meteor., 3, 41-58, https://doi.org/10.15191/nwajom.2015.0305.

Gelaro, R., and Coauthors, 2017: The Modern-Era Retrospective Analysis for Research and Applications, version 2 (MERRA-2). J. Climate, 30, 5419-5454, https://doi.org/10.1175/JCLI-D-160758.1.

Greenwald, T. J., G. L. Stephens, T. H. Vonder Haar, and D. L. Jackson, 1993: A physical retrieval of cloud liquid water over 
the global oceans using Special Sensor Microwave/Imager (SSM/I) observations. J. Geophys. Res., 98, 18 471-18478, https://doi.org/10.1029/93JD00339.

John, V. O., G. Holl, N. Atkinson, and S. A. Buehler, 2013: Monitoring scan asymmetry of microwave humidity sounding channels using simultaneous all angle collocations (SAACs). J. Geophys. Res. Atmos., 118, 1536-154, https://doi.org/ 10.1002/jgrd.50154.

Kazumori, M., and S. J. English, 2015: Use of the ocean surface wind direction signal in microwave radiance assimilation. Quart. J. Roy. Meteor. Soc., 141, 1354-1375, https://doi.org/ 10.1002/qj.2445.

Kulie, M. S., R. Bennartz, T. J. Greenwald, Y. Chen, and F. Weng, 2010: Uncertainties in microwave properties of frozen precipitation: Implications for remote sensing and data assimilation. J. Atmos. Sci., 67, 3471-3487, https://doi.org/10.1175/ 2010JAS3520.1.

Le Vine, D. M., E. P. Dinnat, T. Meissner, S. H. Yueh, F. J. Wentz, S. E. Torrusio, and G. Lagerloef, 2015: Status of Aquarius/ SAC-D and Aquarius salinity retrievals. IEEE J. Sel. Top. Appl. Earth Obs. Remote Sens., 8, 5401-5415, https://doi.org/ 10.1109/JSTARS.2015.2427159.

Liu, G., 2008: A database of microwave single-scattering properties for nonspherical ice particles. Bull. Amer. Meteor. Soc., 89, 1563-1570, https://doi.org/10.1175/2008BAMS2486.1.

Ma, Y., Z. Xiaolei, and F. Weng, 2017: Potential applications of small satellite microwave observations for monitoring and predicting global fast-evolving weathers. IEEE J. Sel. Top. Appl. Earth Obs. Remote Sens., 10, 2441-2551, https://doi.org/ 10.1109/JSTARS.2017.2663335.

Mätzler, C., P. W. Rosenkranz, and J. Cermak, 2010: Microwave absorption of supercooled clouds and implications for the dielectric properties of water. J. Geophys. Res., 115, D23208, https://doi.org/10.1029/2010JD014283.

Mears, C. A., B. D. Santer, F. J. Wentz, K. E. Taylor, and M. F. Wehner, 2007: Relationship between temperature and precipitable water changes over tropical oceans. Geophys. Res. Lett., 34, L24709, https://doi.org/10.1029/2007GL031936.
_ J. Wang, D. Smith, and F. J. Wentz, 2015: Intercomparison of total precipitable water measurements made by satelliteborne microwave radiometers and ground-based GPS instruments. J. Geophys. Res. Atmos., 120, 2492-2504, https:// doi.org/10.1002/2014JD022694.

Nowell, H., G. Liu, and R. Honeyager, 2013: Modeling the microwave single-scattering properties of aggregate snowflakes. J. Geophys. Res. Atmos., 118, 7873-7885, https://doi.org/10.1002/jgrd.50620.

O'Gorman, P. A., and C. J. Muller, 2010: How closely do changes in surface and column water vapor follow Clausius-Clapeyron scaling in climate change simulations? Environ. Res. Lett., 5, 025207, https://doi.org/10.1088/1748-9326/5/2/025207.

Reising, S. C., and Coauthors, 2015: Overview of Temporal Experiment for Storms and Tropical Systems (TEMPEST) CubeSat constellation mission. IEEE MTT-S Int. Microwave Symp., Phoenix, AZ, Institute of Electrical and Electronics Engineers, https://doi.org/10.1109/MWSYM.2015.7167136.

Reynolds, R. W., T. M. Smith, C. Liu, D. B. Chelton, K. S. Casey, and M. G. Schlax, 2007: Daily high-resolution-blended analyses for sea surface temperature. J. Climate, 20, 5473-5496, https://doi.org/10.1175/2007JCLI1824.1.

Rodgers, C. D., 2000: Inverse Methods for Atmospheric Sounding: Theory and Practice. World Science, $240 \mathrm{pp}$.

Wang, J., L. Zhang, A. Dai, T. Van Hove, and J. Van Baelen, 2007: A near-global, 2-hourly atmospheric precipitable water from ground-based GPS measurements. J. Geophys. Res., 112, D11107, https://doi.org/10.1029/2006JD007529.

Ware, R. H., and Coauthors, 2000: SuomiNet: A real-time national GPS network for atmospheric research and education. Bull. Amer. Meteor. Soc., 81, 677-694, https://doi.org/10.1175/15200477(2000)081<0677:SARNGN>2.3.CO;2.

Wentz, F. J., 1997: A well-calibrated ocean algorithm for Special Sensor Microwave/Imager. J. Geophys. Res., 102, 8703-8718, https://doi.org/10.1029/96JC01751.

Wilheit, T. T., and A. T. C. Chang, 1980: An algorithm for retrieval of ocean's surface and atmospheric parameters from the observations of the Scanning Multichannel Microwave Radiometer. Radio Sci., 15, 525-544, https://doi.org/10.1029/RS015i003p00525. 